\title{
Implementación del sistema del último planificador en el sector constructor colombiano: Caso de estudio
}

\author{
Implementation of the last planner system in colombian \\ construction sector: A case study \\ Maria Fernanda Hoyos Restrepo ${ }^{1} \quad$ Luis Fernando Botero Botero ${ }^{1 *}$ \\ Recibido 20 de Junio de 2019, aceptado 3 de mayo de 2021 \\ Received: June 20, 2019 Accepted: May 3, 2021
}

\begin{abstract}
RESUMEN
La filosofía de la construcción sin pérdidas ha sido acogida por numerosas compañías en Colombia desde hace poco más de una década. Entre las herramientas aplicadas se encuentra el Sistema del Último Planificador (SUP) o Last Planner System (LPS), sistema de planificación y control que busca encontrar un flujo de trabajo continuo. El resultado de la implementación del sistema varía de una compañía a otra y no hay un trabajo de investigación que a la fecha exponga un diagnóstico de la aplicación de la herramienta en el país. Este artículo pretende ilustrar el grado de implementación y funcionamiento del SUP desde la perspectiva de dieciséis empresas constructoras colombianas. La recolección de la información se llevó a cabo mediante tres técnicas empleadas en la investigación social cualitativa: La observación no participante, la encuesta y la entrevista semiestructurada. La observación no participante se llevó a cabo en el marco de la reunión de planificación semanal de los proyectos de las empresas constructoras participantes, la encuesta se aplicó a tres grupos: Profesionales de obra, maestros y subcontratistas, y personal operativo; finalmente la entrevista se llevó a cabo con los encargados del área Lean en las compañías. La compilación, triangulación y análisis de la información muestra como resultado múltiples formas de aplicar la herramienta, algunas fallas conceptuales y prácticas, así como falta de entrenamiento y retroalimentación permanente que limita la apropiación del SUP como parte de la cultura empresarial.
\end{abstract}

Palabras clave: Colombia, construcción sin pérdidas, planificación, sistema del último planificador.

\begin{abstract}
The Lean construction philosophy has been welcomed by numerous companies in Colombia for a little more than a decade. The Last Planner System (LPS) is among the implemented tools found, a planning and control system that seeks to accomplish a continuous workflow. The system implementation results vary from one company to another, and there is no investigation to date that shows the diagnosis of the adoption of this tool in the country. This paper aims to illustrate the degree of implementation and functioning of the LPS from different perspectives found in sixteen Colombian construction companies. Data collection was carried out through non-participant observation during the weekly planning meetings, surveys conducted to construction professionals, supervisors, subcontractors and labor, and a semi-structured direct interview with the managers of the Lean area in the companies. Analysis of compiled information shows multiple ways of applying this tool, conceptual and practical failures, and a lack of training and permanent feedback that does not allow to be a part of the corporate culture were all identified.
\end{abstract}

Keywords: Colombia, last planner system, lean construction, planning.

\footnotetext{
1 Universidad EAFIT. Departamento de Ingeniería civil. Medellín, Colombia.

E-mail:mhoyosr3@eafit.edu.co; lfbotero@eafit.edu.co

* Autor de correspondencia: 1fbotero@eafit.edu.co
} 


\section{INTRODUCCIÓN}

El proceso de planificación en las firmas constructoras, y en general, en cualquier tipo de empresa, resulta ser uno de los factores más influyentes en la consecución de los objetivos trazados; un proceso de planificación acertado impacta positivamente en la eficacia con la que se lleva a cabo cada etapa del proyecto y, por ende, repercute en el éxito del mismo. El gremio constructor, en particular, se ha visto afectado por algunas variables que dificultan el flujo continuo de trabajo: Incertidumbre, variabilidad e imprevistos; especialmente dentro de la etapa constructiva de los proyectos. Desde los enfoques de gestión, se han desarrollado diferentes herramientas que pretenden disminuir dichos aspectos y mitigar los impactos negativos que estos generan. Se destaca en particular la filosofía de producción Lean adaptada al sector constructor, que enfatiza en la generación de valor y la disminución de las pérdidas a partir de la eficiencia de los procesos. Dicha filosofía se originó dentro de los principios del sistema de producción impulsado por la compañía Toyota desde la década de 1950 y se ajustó al sector de la construcción en 1992, a partir de la publicación del profesor Lauri Koskela [1] "Aplication of the new production philosophy to construction". El término Lean se traduce de forma literal como "esbelto" o "ajustado", puesto que alude a un sistema de producción que usa una menor cantidad de recursos para producir una mayor y creciente variedad de productos, agregar valor y eliminar pérdidas y defectos en los mismos.

$\mathrm{Al}$ respecto, como uno de los hitos más efectivos en la construcción Lean, se destaca el Last Planner System (LPS) o Sistema del Último Planificador (SUP), ideado por los profesores Gregory Howell y Glenn Ballard en 1992, el cual propone un sistema de planificación y control de la producción diseñado para generar un flujo de trabajo predecible y un aprendizaje rápido en programación, diseño, construcción y puesta en marcha de proyectos. Con el SUP los procesos se llevan a cabo de manera más transparente y se disminuye la variabilidad e incertidumbre características de la construcción, aumentando la confiabilidad de las tareas, definidas como "asignaciones" [2]. La formulación de dichas asignaciones es el resultado de integrar lo que se debe hacer, dentro de las limitaciones de lo que se puede hacer, para decidir lo que se hará y finalmente evaluar lo que se hizo. El qué debe hacerse se concibe desde el plan maestro o plan general que dirige el proyecto, donde se determinan restricciones, duraciones y resultados de cada actividad individual, y se programa una red lógica de actividades a largo plazo. El qué puede hacerse se estudia desde el plan intermedio o plan a mediano plazo, en el que se reduce la incertidumbre en la producción al analizar que cada bloque de actividades cuente con los recursos requeridos para ser ejecutado y que no haya restricciones que limiten su inicio oportuno. El qué se hará se define en el plan semanal o plan a corto plazo, que consiste en un proceso colaborativo con los subcontratistas, donde se establecen acuerdos y objetivos acerca de las tareas a realizar en el transcurso de la semana siguiente, asignando un responsable para cada actividad y verificando si todas las restricciones han sido liberadas. Finalmente, lo que se hizo permite conocer qué porcentaje de las actividades planificadas fueron completadas y cuáles fueron las causas de incumplimiento, con el fin de retroalimentar al grupo de trabajo y promover el mejoramiento continuo.

La planificación vista desde un enfoque Lean a través del SUP, reduce la incertidumbre dentro del proceso de planificación, pues incluye, previo a la ejecución de las actividades, el análisis de restricciones en el plan intermedio que permiten asegurar que las asignaciones puedan ser ejecutadas y retroalimenta semanalmente el proceso para que haya aprendizaje dentro del equipo de trabajo y se concentren esfuerzos por identificar la causa raíz de los incumplimientos para evitar recurrencia futura. El proceso de planificación debe centrarse principalmente en la gestión del puede; mientras más se pueda agrandar el puede, mayor será la posibilidad real de avance, pues si la cantidad de actividades que pueden ejecutarse es baja, el ritmo de avance puede verse afectado [3].

El cambio de paradigma en el sector de la construcción en Colombia ha sido progresivo y el panorama es alentador, pues hoy por hoy las buenas prácticas se están volviendo hábito y la filosofía Lean, asistida por las metodologías como el SUP, está impactando de manera positiva. Sin embargo, se necesita una mayor producción académica que particularice a nivel nacional el panorama del sector construcctor y que permita crear una base teórica que fundamente la construcción Lean, adaptada 
a nuestro entorno. Con base en lo anterior, surge para los autores la motivación de ahondar en la exploración del grado de implementación del SUP en Colombia, el efecto generado por la herramienta y el grado de conocimiento de los profesionales y demás involucrados en los procesos constructivos de las compañías, pues en un futuro la aspiración es tener un gremio integrado, sin baches entre diseño, planificación y construcción, de modo que el producto final desde su concepción hasta su entrega sea trabajado eficientemente y satisfaga las expectativas de valor del cliente.

\section{CONTEXTO DE LA ACTIVIDAD EDIFICADORA COLOMBIANA}

La actividad de la construcción ha sido un componente significativo dentro de la economía colombiana durante las últimas décadas. Desde comienzos del siglo XXI, el valor de la producción de la construcción de edificaciones mantuvo una tendencia creciente, intensificada entre 2012 y 2016, con un crecimiento promedio anual del $10 \%$. Durante el 2017 se dio una contracción en el sector, debido a la desaceleración de la economía nacional, los efectos de la caída de los ingresos petroleros y el incremento del costo del crédito. Pese a que en 2018 aún existían rezagos de esta contracción, las cifras que dinamizó el sector fueron muy significativas; según la Cámara Colombiana de la Construcción (CAMACOL), a partir del año 2019 se espera una estabilización de los indicadores líderes de vivienda y una recuperación más contundente [4].

Los datos recientes del Departamento Administrativo Nacional de Estadísticas (DANE), indican que durante el tercer trimestre de 2018, el PIB colombiano a precios constantes aumentó $2,7 \%$ con relación al mismo trimestre de 2017; el valor agregado del sector constructor aumentó un $1,8 \%$, producto del aumento de $4,1 \%$ y $1,3 \%$ en el subsector de construcción de edificaciones residenciales y actividades especializadas, respectivamente [5].

Respecto al mercado laboral, durante el trimestre móvil octubre - diciembre de 2018, el número de ocupados en el total nacional fue 22.8 millones de personas y la construcción, como rama de actividad económica, participó con el $6.4 \%$ de los ocupados, es decir 1.46 millones de personas. De estos, el 87,0\% estaban ubicados en las cabeceras ( 1.27 millones de personas) y el $13,0 \%$ ( 0,19 millones de personas) en centros poblados y rural disperso [5].

\section{EL SUP: BARRERAS Y BENEFICIOS DE SU IMPLEMENTACIÓN}

El Sistema del Último Planificador es un sistema de planificación y control de la producción basado en la filosofía de construcción sin pérdidas, cuyo objetivo es disminuir la incertidumbre y variabilidad en el flujo de trabajo. El enfoque de la metodología en un principio era el mejoramiento de la calidad de las asignaciones en los planes de trabajo semanales, posteriormente se añadió el proceso lookahead para dar forma y control al flujo de trabajo, y finalmente se extendió de la construcción al diseño. El objetivo migró del mejoramiento de la productividad al mejoramiento de la confiabilidad del flujo de trabajo [2]. Dentro del marco teórico del SUP se reconocen cuatro niveles de planificación que van desde lo general a lo particular: Planificación general, planificación de fases, planificación intermedia o lookahead y planificación a corto plazo. En Colombia, sin embargo, se hace énfasis en la planificación general, planificación intermedia y planificación a corto plazo.

La planificación general, a largo plazo o plan maestro, corresponde al primer eslabón en la cadena jerárquica del SUP; en esta etapa se definen, a grandes rasgos, los capítulos y subcapítulos que componen el proyecto, se establecen las fechas de comienzo y fin de las actividades propias de cada subcapítulo y la relación de dependencia entre las mismas, además, se precisa la estrategia de ejecución del proyecto, la secuencia de actividades y el ritmo al cual se ejecutarán. En la planificación de fases, a través de un trabajo colaborativo, se subdividen los paquetes de actividades del plan general en componentes más detalladas, se especifican los entregables y la fecha de entrega. En la planificación intermedia o lookahead se busca reducir la incertidumbre del proceso constructivo, al determinar con anticipación las restricciones de las actividades, para eliminarlas y asegurar vía libre para la ejecución e inicio oportuno de las asignaciones. Finalmente, el último eslabón de la cadena corresponde a la planificación a corto plazo o planificación semanal, proceso colaborativo en el que participan los subcontratistas, maestros, profesionales de la obra y personal de soporte como el almacenista o el encargado de seguridad y salud en 
el trabajo. Generalmente se lleva a cabo a través de una reunión semanal, donde el equipo mencionado se enfoca en dos aspectos: La evaluación del trabajo concertado para la semana anterior, y la programación de las asignaciones para la semana siguiente, donde además de fijar fechas de comienzo y fin, se define el porcentaje específico de la actividad a realizar y el responsable de esta; además, se discute acerca de la disponibilidad de recursos materiales y humanos en las unidades de actuación.

Los indicadores principales obtenidos con la implementación del SUP son el Porcentaje de Asignaciones Completadas (PAC) y las Causas de No Cumplimiento (CNC). El PAC, relación entre número de asignaciones completadas y el número de asignaciones planificadas, mide el desempeño del proyecto y la confiabilidad del flujo de trabajo [6], puesto que permite ejercer el control a nivel de la unidad de producción [2]. Las CNC, son las razones, generalmente expresadas con porcentajes, que generan los incumplimientos y se pueden clasificar en agentes internos y agentes externos, según su origen. Los agentes internos corresponden a aspectos organizacionales y factores humanos controlables por el personal que administra la obra; los agentes externos son aquellos ajenos al control de la organización [7]. De publicaciones anteriores sobre casos de estudio alrededor del mundo, se traen a colación diferentes barreras encontradas en la implementación del SUP, ocasionadas por factores humanos o culturales y de tipo organizacional, además, resultados significativos que sobresalen en las compañías luego de implementar el sistema, temas abordados en el presente caso de estudio.

Las barreras que han dificultado la implementación del SUP giran en su mayoría en torno a factores humanos o culturales [7]. Se ha destacado el clima de incertidumbre y poca familiaridad con el sistema $[8$, 9]; la dificultad para decir "no" por temor a violar el sistema tradicional basado en el modelo de mando de directivos a subordinados [6, 10]; la falta de entrenamiento y la autocrítica [11, 12]; la visión cortoplacista $[12,13]$; la resistencia al cambio bien sea por miedo, por evitar asumir compromisos o por el deber de incluir a los subcontratistas en las reuniones de planificación [12, 14, 15]; la falta de compromiso y actitud frente a la puntualidad [13]; el escepticismo frente a los beneficios de la implementación [16]; la dificultad para mantener a todos anotando sus retrasos pues la revisión del trabajo ejecutado no era una práctica común [17]; la falta de disposición para asumir compromisos [14]; los inconvenientes con el idioma y la cultura cuando los trabajadores provenían de varias regiones o agrupaciones étnicas diferentes [18-20]; la resistencia de las personas a estar en una reunion por un tiempo prolongado [21] y la dificultad para pasar de dar órdenes a los subcontratistas a motivarlos a asumir sus propios compromisos con las actividades [22]. Se han reportado también barreras de tipo organizacional como la dificultad para construir reservas de actividades ejecutables [9]; la aparente necesidad de resolver problemas en lugar de planificar para evitarlos en el proyecto [6, 10]; la insuficiente preparación de las reuniones de planificación [12]; la implementación parcial o intermitente $[12,23]$; la falta de incentivos para los subcontratistas [24]; la falta de integración de la cadena de producción: Cliente, proveedores de materiales, subcontratistas [11, 18]; la falta de vinculación entre los niveles de planificación del sistema, lo cual disminuye el enfoque proactivo y ocasiona que el PAC pierda relación con el progreso general del proyecto [25, 26]; la falta de comunicación y de gerenciamiento visual para generar información oportuna [14]; el bajo entendimiento de los elementos y conceptos del SUP: El por qué debe ser usado y los beneficios que trae su implementación [11, 18]; la falta de entendimiento de las necesidades del proyecto por parte de los subcontratistas [24]; la forma de contratación, pues generalmente el diseño se contrata independiente a la construcción, lo cual obstaculiza la integración del conocimiento e incide en la recurrencia de cambios futuros [17]; el exceso de compromisos sobre el trabajo que puede hacerse desde la planificación intermedia [18].

Respecto a los resultados más relevantes obtenidos con la implementación del SUP el más común ha sido la mejora en el PAC, reflejo del aumento de la confiabilidad del sistema de planificación [6, 9-12, 16, 27-33]; asimismo se han divulgado resultados como la mejora en el factor de productividad, el cual indica la relación entre los rendimientos reales y presupuestados $[6,9,29]$; la disminución de reprocesos [34, 35]; la mejor distribución de los recursos en el tiempo [34]; la disminución de la duración total del proyecto $[11,17,34]$; una mejor organización in situ $[34,36]$; la reducción en la variabilidad del PAC $[11,12,37]$; el incremento 
en la productividad de la mano de obra [11]; la eliminación de los tiempos de espera [35]; el apoyo de alta dirección y la participación de todos las partes interesadas [13]; la colaboración y coordinación entre los miembros del equipo [13, $15,20,38]$; una mayor transparencia en el proceso de planificación [36]; un mayor control sobre las asignaciones y productividad del subcontratista [36, 38]; la disminución en la variación de la duración de las actividades con holguras más ajustadas [39, 40] y el apoyo de la gerencia y la definición de roles y responsabilidades [20].

\section{METODOLOGÍA DE INVESTIGACIÓN}

El trabajo de investigación se enmarca principalmente dentro del enfoque cualitativo, a partir del cual se estudió la dimensión interna y particular de los actores sociales, se permeó en su cultura al vivirla desde adentro y observar de forma pasiva su comportamiento. Se generaron datos descriptivos y datos que posteriormente se codificaron con el fin de ser cuantificados por medio de herramientas estadísticas simples para obtener indicadores, reconocer tendencias e identificar patrones de comportamiento entre las compañías constructoras. Los procedimientos de codificación ofrecen a los investigadores herramientas útiles para manejar grandes cantidades de datos brutos, ayudan a considerar significados alternativos de los fenómenos y permiten identificar, desarrollar y relacionar los conceptos [41].

\section{Recolección de la información}

La recolección de la información puede categorizarse como el eje central del proceso de investigación social cualitativa, pues es el fundamento a partir del cual el investigador da respuesta a los interrogantes que motivan el trabajo. En este caso se emplearon tres técnicas para la recolección de la información: La observación no participante, la entrevista semiestructurada y la encuesta.

El trabajo de campo se desarrolló con la colaboración de 16 empresas constructoras colombianas que aplican los principios de la construcción sin pérdidas y el SUP como su sistema de planificación y control. A pesar de que la muestra no es representativa de todas las constructoras colombianas de edificaciones, sí lo es del grupo de empresas que se han esforzado por conocer los principios y conceptos de la filosofía
Lean y que han dado un paso a la implementación al interior de sus proyectos, pues en el sector nacional aún es muy incipiente la filosofía de construcción sin pérdidas pese a los esfuerzos realizados por algunos establecimientos de educación superior en la capacitación del sector.

Los autores seleccionaron la encuesta con el fin de conocer el punto de vista de aquellos que aplican en sitio el SUP, la entrevista con el objetivo de conocer la opinión de aquellos encargados de dirigir el área Lean en las compañías, y la observación no participante como una forma de triangulación de la información obtenida para enriquecer y fortalecer la credibilidad de los datos obtenidos. En la Tabla 1 se presenta el resumen de las empresas colaboradoras y en la Tabla 2 se muestran los instrumentos para la recolección de la información usados en cada una de ellas.

\section{Observación no participante}

La observación no participante es aquella donde el observador realiza su trabajo permaneciendo ajeno al grupo, sin involucrarse o interferir en su cotidianidad. Obedece a un proceso estructurado, pero pretende realizar un acercamiento más objetivo a la comunidad o grupo de estudio, sin implicarse de manera directa [42]. El observador, en esta situación actúa más como un espectador que como un actor; el distanciamiento tiene la ventaja de la objetividad del punto de vista del observador, sin embargo, se puede correr el riesgo de que durante el desarrollo del ejercicio solo se obtengan datos superficiales [43].

El proceso de observación no participante se realizó durante las reuniones de planificación semanal de 33 obras de construcción: 23 en el departamento de Antioquia, 6 en Cundinamarca, 2 en Caldas y 2 en Santander. Se visitaron entre 1 y 4 obras en cada compañía constructora, según la disponibilidad de cada una, con una duración promedio de reunión por obra de noventa minutos. Con el fin de delimitar la información más relevante y adoptar un criterio común para sistematizar y codificar los datos encontrados desde la posición de observador, se elaboró una guía para el registro de información (ver Tabla 3), dividida en 3 secciones principales: 1) desarrollo de la reunión, 2) formalidad en la reunión y 3 ) calificación del desempeño a subcontratistas. 
Tabla 1. Empresas constructoras colaboradoras.

\begin{tabular}{|c|c|c|c|c|}
\hline Constructora & Antigüedad & $\begin{array}{l}\text { Tipo de } \\
\text { operación }\end{array}$ & Sitio de operación & Nicho de mercado actual \\
\hline A & 67 años & Nacional & $\begin{array}{l}\text { Antioquia, Bolívar, Cundinamarca, } \\
\text { Magdalena, Quindío, Valle del Cauca }\end{array}$ & $\begin{array}{l}\text { Centros de salud, comercio, hotelería, } \\
\text { industrial, infraestructura, institucional, } \\
\text { oficinas, vivienda }\end{array}$ \\
\hline $\mathrm{B}$ & 71 años & Regional & Antioquia & Bodegas, industrial, institucional, vivienda \\
\hline $\mathrm{C}$ & 26 años & Nacional & Antioquia, Atlántico, Cundinamarca & $\begin{array}{l}\text { Centros de salud, comercio, institucional, } \\
\text { vivienda }\end{array}$ \\
\hline $\mathrm{D}$ & 23 años & Nacional & Antioquia, Cundinamarca & Centros de salud, comercio, vivienda \\
\hline $\mathrm{E}$ & 17 años & Nacional & $\begin{array}{l}\text { Caldas, Cundinamarca, Huila, Risaralda, } \\
\text { Valle del Cauca }\end{array}$ & Oficinas, vivienda \\
\hline $\mathrm{F}$ & 52 años & \begin{tabular}{|l} 
Nacional e \\
internacional
\end{tabular} & Colombia, Centroamérica & Todo tipo de edificaciones e infraestructura \\
\hline G & 53 años & Regional & Antioquia & Vivienda \\
\hline $\mathrm{H}$ & 25 años & Nacional & Antioquia, Cundinamarca & Vivienda \\
\hline $\mathrm{I}$ & 41 años & Nacional & Cundinamarca, Valle del Cauca & Comercio, vivienda \\
\hline $\mathrm{J}$ & 15 años & Nacional & Cundinamarca, Santander & Vivienda \\
\hline $\mathrm{K}$ & 27 años & Nacional & Antioquia, Cundinamarca, Sucre & Comercio, institucional, oficinas, vivienda \\
\hline $\mathrm{L}$ & 10 años & Regional & Antioquia & Hotelería, Oficinas, vivienda \\
\hline M & 26 años & $\begin{array}{l}\text { Nacional e } \\
\text { internacional }\end{array}$ & Colombia, Estados Unidos & Comercio, vivienda \\
\hline $\mathrm{N}$ & 24 años & Nacional & $\begin{array}{l}\text { Cundinamarca, Norte de Santander, } \\
\text { Tolima, Valle del Cauca }\end{array}$ & Oficinas, vivienda \\
\hline $\mathrm{O}$ & 6 años & Nacional & $\begin{array}{l}\text { Atlántico, Bolívar, Cundinamarca, Valle } \\
\text { del Cauca }\end{array}$ & Bodegas, call center, data center, industrial \\
\hline $\mathrm{P}$ & 16 años & Regional & Norte de Santander & Vivienda \\
\hline
\end{tabular}

Tabla 2. Elementos para la recolección de la información utilizados.

\begin{tabular}{|c|c|c|c|c|c|}
\hline Constructora & $\begin{array}{c}\text { Observación } \\
\text { no participante }\end{array}$ & $\begin{array}{c}\text { Encuesta a } \\
\text { profesionales de obra }\end{array}$ & $\begin{array}{c}\text { Encuesta a maestros } \\
\text { y subcontratistas }\end{array}$ & $\begin{array}{c}\text { Encuesta a } \\
\text { personal operativo }\end{array}$ & Entrevista \\
\hline $\mathrm{A}$ & $\mathrm{x}$ & $\mathrm{x}$ & $\mathrm{x}$ & $\mathrm{x}$ & $\mathrm{x}$ \\
\hline B & $\mathrm{x}$ & & $\mathrm{x}$ & $\mathrm{x}$ & $\mathrm{x}$ \\
\hline $\mathrm{C}$ & $\mathrm{x}$ & & $\mathrm{x}$ & $\mathrm{x}$ & $\mathrm{x}$ \\
\hline $\mathrm{D}$ & $\mathrm{x}$ & $\mathrm{x}$ & $\mathrm{x}$ & $\mathrm{x}$ & $\mathrm{x}$ \\
\hline $\mathrm{E}$ & $\mathrm{x}$ & $\mathrm{x}$ & $\mathrm{x}$ & $\mathrm{x}$ & $\mathrm{x}$ \\
\hline $\mathrm{F}$ & $\mathrm{x}$ & $\mathrm{x}$ & $\mathrm{x}$ & $\mathrm{x}$ & $\mathrm{x}$ \\
\hline $\mathrm{G}$ & $\mathrm{x}$ & & $\mathrm{x}$ & $\mathrm{x}$ & $\mathrm{x}$ \\
\hline $\mathrm{H}$ & $\mathrm{x}$ & $\mathrm{x}$ & $\mathrm{x}$ & $\mathrm{x}$ & $\mathrm{x}$ \\
\hline $\mathrm{I}$ & $\mathrm{x}$ & $\mathrm{x}$ & $\mathrm{x}$ & $\mathrm{x}$ & $\mathrm{x}$ \\
\hline $\mathrm{J}$ & $\mathrm{x}$ & $\mathrm{x}$ & $\mathrm{x}$ & $\mathrm{x}$ & $\mathrm{x}$ \\
\hline $\mathrm{K}$ & $\mathrm{x}$ & & $\mathrm{x}$ & $\mathrm{x}$ & $\mathrm{x}$ \\
\hline $\mathrm{L}$ & $\mathrm{x}$ & $\mathrm{x}$ & $\mathrm{x}$ & $\mathrm{x}$ & $\mathrm{x}$ \\
\hline M & $\mathrm{x}$ & $\mathrm{x}$ & $\mathrm{x}$ & $\mathrm{x}$ & $\mathrm{x}$ \\
\hline $\mathrm{N}$ & $\mathrm{x}$ & $\mathrm{x}$ & $\mathrm{x}$ & $\mathrm{x}$ & $\mathrm{x}$ \\
\hline $\mathrm{O}$ & & $\mathrm{x}$ & $\mathrm{x}$ & $\mathrm{x}$ & $\mathrm{x}$ \\
\hline $\mathrm{P}$ & & $\mathrm{x}$ & $\mathrm{x}$ & $\mathrm{x}$ & \\
\hline
\end{tabular}


Tabla 3. Guía para el registro de información en la observación no participante.

\begin{tabular}{|c|c|c|c|c|}
\hline Sección & Parámetro observado & & Anotación & \\
\hline \multirow{7}{*}{$\begin{array}{l}\text { Desarrollo } \\
\text { de la reunión }\end{array}$} & $\begin{array}{l}\text { ¿Al comenzar la reunión existe una calificación previa } \\
\text { de las actividades ejecutadas por el subcontratista? }\end{array}$ & Sí [ & NO & \\
\hline & $\begin{array}{l}\text { ¿Existe un listado detallado de las CNC definidas } \\
\text { en la obra? }\end{array}$ & Sí [ & NOL & \\
\hline & ¿Hay discusión sobre las CNC? & Sí [ & NOL & \\
\hline & ¿El PAC se registra con 0 y 1 ? & Sí & $\mathrm{NO}$ & \\
\hline & $\begin{array}{l}\text { ¿Hay registro de la programación para la semana } \\
\text { siguiente? }\end{array}$ & Síl & $\mathrm{NO}$ & \\
\hline & ¿Hay claridad sobre las tareas a programar? & Sí [ & $\mathrm{NO}$ & \\
\hline & Calificación de la participación de los subcontratistas & 0[ & 0,5 & 1 \\
\hline \multirow{6}{*}{$\begin{array}{l}\text { Formalidad } \\
\text { de la reunión }\end{array}$} & ¿Hay un orden del día establecido? & Sí [ & $\mathrm{NO}$ & \\
\hline & ¿La reunión se programa rutinariamente? & Sí [ & $\mathrm{NO}$ & \\
\hline & ¿Se toma lista de asistencia? & Sí [ & $\mathrm{NO}$ & \\
\hline & ¿Se entrega la programación de obra al personal? & Sí [ & NOL & \\
\hline & ¿Se firman los compromisos? & Sí & $\mathrm{NO}$ & \\
\hline & Calificación del orden & 0[ & $0,5 \square$ & 1[ \\
\hline \multirow{4}{*}{$\begin{array}{l}\text { Calificación a } \\
\text { subcontratistas }\end{array}$} & $\begin{array}{l}\text { ¿Se socializa la calificación semanal de los subcontratistas } \\
\text { obtenida según el cumplimiento de las asignaciones? }\end{array}$ & Síl & $\mathrm{NO}$ & \\
\hline & $\begin{array}{l}\text { ¿Se evalúan todas las variables empleadas para calificar } \\
\text { el desempeño de subcontratistas? }\end{array}$ & sí [ & $\mathrm{NO} \square$ & \\
\hline & ¿La calificación de las variables es cualitativa o cuantitativa? & Cualitativa & Cualitativa & \\
\hline & $\begin{array}{l}\text { ¿Existe cartelera de desempeño para calificar a los } \\
\text { subcontratistas? }\end{array}$ & sí[ & NO & \\
\hline
\end{tabular}

\section{Encuestas}

La encuesta se puede definir como una técnica que consiste en recopilar información sobre una parte de la población denominada muestra [44]; el investigador recoge las respuestas a una serie de preguntas mediante un cuestionario elaborado previamente. La construcción del cuestionario, supone seguir una metodología sustentada en el cuerpo de la teoría, el marco conceptual en que se apoya el estudio, las hipótesis que se pretender probar y los objetivos de la investigación [44].

La información se registró mediante papel y lápiz, paso seguido se digitalizó su contenido, transcribiendo las respuestas de forma literal, posteriormente se tabularon dichas respuestas y, aquellas abiertas, se agruparon y categorizaron. Se realizaron tres tipos de encuesta, con algunas preguntas en común, pero conservando un enfoque diferente según el sector de la muestra objetivo. Los resultados se agruparon en cuatro ejes temáticos: 1) noción de la herramienta, 2) aspectos generales de planificación y control, 3) aspectos del componente humano del sistema y 4) apreciaciones de la herramienta.

El primer tipo de encuesta se dirigió a maestros de obra y subcontratistas, quienes representan el último eslabón en la cadena de planificación. El segundo tipo de encuesta se orientó al personal operativo, es decir, la mano de obra directa que interviene en el proceso constructivo. Finalmente, el tercer tipo de encuesta dirigió a los profesionales de la obra: Coordinadores, directores y residentes de obra. Se realizaron en total 392 encuestas: 183 encuestas tipo 1, 122 encuestas tipo 2 y 83 encuestas tipo 3 . En la Tabla 4 se presenta la encuesta tipo 3.

\section{Entrevistas}

La entrevista puede definirse como una estrategia para hacer que la gente hable sobre lo que sabe, piensa y cree, una situación en la cual una persona 
Tabla 4. Encuesta dirigida a los profesionales de obra.

\begin{tabular}{|c|c|c|c|c|c|c|}
\hline Pregunta & Respuest & & & & & \\
\hline $\begin{array}{l}\text { ¿Sabe usted qué es el LAST PLANNER o Sistema } \\
\text { del Último Planificador? }\end{array}$ & Sí $\square$ & $\mathrm{NO} \square$ & & & & \\
\hline $\begin{array}{l}\text { Si la respuesta anterior es "SÍ", ¿Qué es para } \\
\text { usted el LAST PLANNER o Sistema del Último } \\
\text { Planificador? }\end{array}$ & & & & & & \\
\hline $\begin{array}{l}\text { ¿Tuvo usted alguna capacitación inicial sobre el } \\
\text { sistema de planificación que se usa en la obra? }\end{array}$ & Sí & NOL & & & & \\
\hline Si la respuesta anterior es "SÍ", ¿Cómo se capacitó? & & & & & & \\
\hline $\begin{array}{l}\text { ¿Se hace benchmarking entre obras de la empresa } \\
\text { o proyectos de otras compañías? }\end{array}$ & Sí [ & NO $\square$ & & & & \\
\hline ¿Conoce usted la programación general de la obra? & Sí [ & NO & & & & \\
\hline ¿Con qué software se hace esta programación? & & & & & & \\
\hline $\begin{array}{l}\text { ¿Cuál es el horizonte de tiempo de la planificación } \\
\text { intermedia? }\end{array}$ & & & & & & \\
\hline ¿Llevan registro de las restricciones? & Sí [ & NOL & & & & \\
\hline $\begin{array}{l}\text { ¿Con qué periodicidad se llevan a cabo las reuniones } \\
\text { de planificación intermedia? }\end{array}$ & & & & & & \\
\hline $\begin{array}{l}\text { ¿Cuántas veces a la semana se reúne con su } \\
\text { equipo de trabajo (director, residentes, maestros, }\end{array}$ & $1 \square$ & & $3 \square$ & $4 \square$ & $5 \square$ & Otro $\square$ \\
\hline trabajo que se ha hecho y el que está por hace & ¿Cada cu & ánto? & & & & \\
\hline $\begin{array}{l}\text { ¿En la reunión se habla sobre la causa de no } \\
\text { cumplimiento de las actividades? }\end{array}$ & Sí & NOL & & & & \\
\hline $\begin{array}{l}\text { ¿Se buscan soluciones para que no vuelvan a } \\
\text { ocurrir las causas de no cumplimiento? }\end{array}$ & SÍ L & NOL & & & & \\
\hline ¿Cuál es la causa más frecuente de no cumplimiento? & & & & & & \\
\hline ¿Cómo cree usted que es el sistema de planificación & Muy bu & & no & Regular [ & & \\
\hline y control actual en la obra? & Malo & Muy m & & & & \\
\hline
\end{tabular}

obtiene información sobre algo interrogando a otra; dicha información suele referirse a la biografía, al sentido de los hechos, a sentimientos, opiniones y emociones, a las normas o estándares de acción, y a los valores o conductas ideales [45]. Particularmente, la entrevista con fines de investigación busca, a través de la información obtenida de un conjunto de saberes privados, la construcción del sentido social de la conducta individual o del grupo de referencia de ese individuo [46].

Los autores realizaron un total de 15 entrevistas abiertas semiestructuradas a los encargados de coordinar el área Lean o productividad en las compañías, cada una con una duración entre treinta y noventa minutos. Se abordó primero la información acerca de la identificación de la empresa, posteriormente se indagó sobre la identificación del empleado y su rol dentro de la compañía, después se ahondó de forma general sobre la implementación de la construcción Lean y finalmente se formularon las preguntas acerca de la implementación específica del SUP, su impacto, la opinión del entrevistado sobre el efecto de la herramienta y los puntos a mejorar. En la Tabla 5 se presenta la guía empleada por los autores para dirigir la entrevista.

\section{ANÁLISIS DE DATOS}

\section{Observación no participante}

Durante el desarrollo de la reunión se prestó especial atención a la existencia de una calificación previa de las actividades ejecutadas por el subcontratista durante la semana, al detalle y discusión acerca de las $\mathrm{CNC}$, la calificación del PAC, la programación 
Tabla 5. Guía para la entrevista con los encargados de dirigir el área Lean en las compañías.

\begin{tabular}{|c|c|}
\hline Sección & Pregunta \\
\hline \multirow{7}{*}{ Implementación Lean } & ¿Hace cuánto se implementó la construcción Lean en la empresa? \\
\hline & ¿Existe personal de soporte en las obras para la implementación de esta filosofía? \\
\hline & ¿Cuáles son sus funciones como encargado de dirigir el área Lean? \\
\hline & ¿La empresa tuvo capacitación inicial?, ¿Cómo se llevó a cabo? \\
\hline & Cuando ingresa personal nuevo, ¿Se capacita? \\
\hline & ¿Hay retroalimentación de las herramientas?, ¿Cada cuánto?, ¿Quiénes participan? \\
\hline & ¿Se hace benchmarking entre obras de la empresa o proyectos de otras compañías? \\
\hline \multirow{16}{*}{$\begin{array}{l}\text { Implementación } \\
\text { del sistema de } \\
\text { planificación y control }\end{array}$} & ¿Hace cuánto se implementó el SUP? \\
\hline & ¿Cómo se capacitó la empresa?, ¿Quiénes recibieron capacitación? \\
\hline & ¿Qué niveles de planificación se han implementado? \\
\hline & ¿Cómo se hace el plan general o plan maestro?, ¿Con qué software? \\
\hline & ¿Cuál es el horizonte de la planificación intermedia?, ¿Cómo se definió? \\
\hline & ¿Con qué periodicidad se llevan a cabo las reuniones de planificación intermedia? \\
\hline & ¿Llevan registro de las restricciones?, ¿Tienen indicadores? \\
\hline & ¿Con qué periodicidad se realizan las reuniones de planificación a corto plazo? \\
\hline & ¿Se exponen las CNC de las actividades? \\
\hline & ¿Se busca la causa raíz que genera el no cumplimiento de las actividades? \\
\hline & ¿Se toman medidas correctivas para que no haya recurrencia de estas CNC? \\
\hline & ¿Los subcontratistas son incluidos en las reuniones de planificación semanal? \\
\hline & ¿Los subcontratistas participan en la toma de decisiones a corto plazo? \\
\hline & ¿Cuál ha sido la mejora más significativa con la implementación del sistema? \\
\hline & ¿Cómo ha sido su percepción del sistema de planificación y control actual en obra? \\
\hline & ¿Qué se podría mejorar a nivel de planificación y control dentro de la obra? \\
\hline
\end{tabular}

de las tareas para la semana siguiente, la claridad de los profesionales acerca de la asignación de actividades y la participación de los subcontratistas. Con el fin de valorar la formalidad en las reuniones, se tuvieron en cuenta aspectos como la existencia de un orden del día, la programación rutinaria de la reunión, la toma de lista o asistencia, la entrega de la programación de obra al personal, la firma de los compromisos adquiridos por parte de los subcontratistas y el orden general durante la reunión. Finalmente, respecto a la calificación integral del subcontratista, se tomó nota sobre la socialización de la calificación durante la reunión, la evaluación de las variables complementarias al PAC para calificar su desempeño y la existencia y características de la cartelera de desempeño. Con el objetivo de poder cuantificar los resultados obtenidos durante el proceso de observación no participante, y facilitar el proceso de análisis y comparación de resultados, los autores asignaron a las preguntas dicotómicas (ver Tabla 3) un 1, si dentro del proyecto se satisfacía el parámetro observado, o un 0 en caso contrario. Para la evaluación de la participación de los subcontratistas se calificó con un 1 si había participación constante, 0,5 cuando la participación era esporádica y 0 si había baja o escasa participación. Respecto al orden durante la reunión, se asignó un 1 si la reunión era organizada, sin improvisación y con el personal atento; $0,5 \mathrm{si}$ la reunión era reunión parcialmente organizada y 0 en el caso de una reunión desorganizada. Se promediaron los resultados de los proyectos visitados por constructora y se expresaron en términos porcentuales, con miras a obtener el promedio total general por parámetro y por compañía.

Es evidente, en términos generales, la falta de rigurosidad especialmente respecto a la discusión y detalle de las CNC. En un $41 \%$ de las compañías no se genera discusión en torno a dicho tema, inclusive hay casos en los cuales no se mencionan, a pesar de tener una lista de chequeo predeterminada en la compañía. En el 59\% restante de los proyectos, sí se genera un espacio de discusión, pero el común denominador es no profundizar en la causa raíz que produce el incumplimiento. Además, el listado de 
las $\mathrm{CNC}$ es muy poco detallado, solo en un $21 \%$ se encontró un listado pormenorizado. Por otra parte, la falta de calificación previa a la reunión de las asignaciones completadas durante la semana y la baja participación de los subcontratistas también son aspectos en los que se debe trabajar en muchas de las constructoras. En la Tabla 6 se muestra el resumen de la evaluación del desarrollo de la reunión semanal clasificado por compañía.

En cuanto a la formalidad durante la reunión, la firma de los compromisos adquiridos para la semana siguiente y la toma de lista o asistencia son los aspectos que tuvieron un mayor número de respuestas negativas. En términos generales, en un 54\% de las obras no se llevaba el registro de asistencia y en un $39 \%$ no se firmaban los compromisos adquiridos. En la mayoría de las obras existe un orden del día, la reunión semanal se programa rutinariamente y se entrega la programación acordada para la semana siguiente a cada subcontratista. El resultado respecto al orden durante el curso de la reunión tuvo resultados muy variables según la constructora. En la Tabla 7 se muestra el resumen de la evaluación de la formalidad de la reunión semanal clasificado por compañía.

Finalmente, referente a la calificación semanal del subcontratista es común encontrar la evaluación de variables complementarias al PAC entre las empresas colombianas que han tenido capacitación acerca del SUP, pues permiten tener una valoración más integral del subcontratista. Estas variables y su ponderación dentro de la evaluación del personal son definidas según el criterio de cada compañía, sin embargo, las más recurrentes son: Calidad, seguridad y salud en el trabajo, orden y aseo, gestión ambiental y recursos. El aspecto más débil en esta sección fue la calificación de dichas variables, pues en la mayoría de las compañías se hace de forma cualitativa, no hay criterios objetivos para su calificación ni un registro del comportamiento de dichas variables en el tiempo. La socialización de la calificación semanal del subcontratista respecto a las asignaciones completadas y la cartelera de desempeño tuvieron respuesta favorable en la mayoría de las empresas. En la Tabla 8 se muestra el resumen de la evaluación de la formalidad de la reunión semanal clasificado por compañía.

\section{Encuestas}

A partir de las encuestas, se encontró que hay una diferencia muy marcada respecto a la noción de la herramienta según el tipo de encuestado (ver Figura 1). De forma general, se observa que en el personal operativo es casi nulo el conocimiento que se tiene acerca del SUP; en los maestros y subcontratistas,

Tabla 6. Evaluación de aspectos relacionados con el desarrollo satisfactorio de la reunión.

\begin{tabular}{|c|c|c|c|c|c|c|c|c|}
\hline Constructora & $\begin{array}{c}\text { Calificación } \\
\text { previa } \\
\text { organizada }\end{array}$ & $\begin{array}{c}\text { Discución } \\
\text { sobre } \\
\text { CNC }\end{array}$ & $\begin{array}{c}\text { Listado } \\
\text { detallado } \\
\text { de } \\
\text { CNC }\end{array}$ & $\begin{array}{c}\text { Registro } \\
\text { del PAC } \\
\text { con 0 } \\
\mathbf{y} \mathbf{1}\end{array}$ & $\begin{array}{c}\text { Registro de la } \\
\text { programación } \\
\text { para la semana } \\
\text { siguiente }\end{array}$ & $\begin{array}{c}\text { Claridad } \\
\text { acerca de } \\
\text { actividades a } \\
\text { programar }\end{array}$ & $\begin{array}{c}\text { Participación } \\
\text { de los } \\
\text { subcontratistas }\end{array}$ & Promedio \\
\hline A & $0 \%$ & $0 \%$ & $0 \%$ & $33 \%$ & $100 \%$ & $33 \%$ & $17 \%$ & $26 \%$ \\
\hline B & $50 \%$ & $50 \%$ & $0 \%$ & $100 \%$ & $100 \%$ & $100 \%$ & $50 \%$ & $64 \%$ \\
\hline C & $0 \%$ & $50 \%$ & $100 \%$ & $100 \%$ & $25 \%$ & $25 \%$ & $50 \%$ & $50 \%$ \\
\hline D & $100 \%$ & $0 \%$ & $0 \%$ & $100 \%$ & $100 \%$ & $100 \%$ & $50 \%$ & $64 \%$ \\
\hline E & $0 \%$ & $0 \%$ & $0 \%$ & $100 \%$ & $100 \%$ & $100 \%$ & $50 \%$ & $50 \%$ \\
\hline F & $100 \%$ & $100 \%$ & $0 \%$ & $100 \%$ & $100 \%$ & $100 \%$ & $100 \%$ & $86 \%$ \\
\hline G & $75 \%$ & $25 \%$ & $100 \%$ & $100 \%$ & $75 \%$ & $100 \%$ & $38 \%$ & $73 \%$ \\
\hline H & $33 \%$ & $0 \%$ & $0 \%$ & $33 \%$ & $100 \%$ & $33 \%$ & $0 \%$ & $29 \%$ \\
\hline I & $100 \%$ & $100 \%$ & $0 \%$ & $100 \%$ & $100 \%$ & $100 \%$ & $100 \%$ & $86 \%$ \\
\hline J & $0 \%$ & $100 \%$ & $0 \%$ & $50 \%$ & $100 \%$ & $100 \%$ & $50 \%$ & $57 \%$ \\
\hline K & $100 \%$ & $100 \%$ & $0 \%$ & $100 \%$ & $33 \%$ & $100 \%$ & $100 \%$ & $76 \%$ \\
\hline L & $100 \%$ & $100 \%$ & $0 \%$ & $100 \%$ & $100 \%$ & $100 \%$ & $100 \%$ & $86 \%$ \\
\hline M & $100 \%$ & $100 \%$ & $100 \%$ & $100 \%$ & $100 \%$ & $100 \%$ & $100 \%$ & $100 \%$ \\
\hline N & $100 \%$ & $100 \%$ & $0 \%$ & $100 \%$ & $100 \%$ & $100 \%$ & $100 \%$ & $86 \%$ \\
\hline General & $61 \%$ & $59 \%$ & $21 \%$ & $87 \%$ & $88 \%$ & $85 \%$ & $65 \%$ & $67 \%$ \\
\hline
\end{tabular}


Tabla 7. Evaluación de aspectos relacionados la formalidad de la reunión.

\begin{tabular}{|c|c|c|c|c|c|c|c|}
\hline Constructora & $\begin{array}{c}\text { Existencia } \\
\text { de agenda }\end{array}$ & $\begin{array}{c}\text { Reunión } \\
\text { programada } \\
\text { rutinariamente }\end{array}$ & $\begin{array}{c}\text { Registro de } \\
\text { asistencia }\end{array}$ & $\begin{array}{c}\text { Entrega de } \\
\text { programación a } \\
\text { subcontratistas }\end{array}$ & $\begin{array}{c}\text { Firma de } \\
\text { compromisos }\end{array}$ & $\begin{array}{c}\text { Orden en } \\
\text { la reunión }\end{array}$ & Promedio \\
\hline A & $33 \%$ & $67 \%$ & $33 \%$ & $33 \%$ & $33 \%$ & $33 \%$ & $39 \%$ \\
\hline B & $100 \%$ & $100 \%$ & $50 \%$ & $100 \%$ & $100 \%$ & $50 \%$ & $83 \%$ \\
\hline C & $100 \%$ & $100 \%$ & $50 \%$ & $75 \%$ & $0 \%$ & $63 \%$ & $65 \%$ \\
\hline D & $100 \%$ & $100 \%$ & $100 \%$ & $100 \%$ & $100 \%$ & $100 \%$ & $100 \%$ \\
\hline E & $100 \%$ & $100 \%$ & $100 \%$ & $100 \%$ & $100 \%$ & $75 \%$ & $96 \%$ \\
\hline F & $100 \%$ & $100 \%$ & $100 \%$ & $100 \%$ & $100 \%$ & $100 \%$ & $100 \%$ \\
\hline G & $100 \%$ & $100 \%$ & $25 \%$ & $100 \%$ & $75 \%$ & $38 \%$ & $73 \%$ \\
\hline H & $100 \%$ & $100 \%$ & $33 \%$ & $33 \%$ & $33 \%$ & $17 \%$ & $53 \%$ \\
\hline I & $100 \%$ & $100 \%$ & $100 \%$ & $100 \%$ & $0 \%$ & $100 \%$ & $83 \%$ \\
\hline J & $100 \%$ & $100 \%$ & $0 \%$ & $50 \%$ & $50 \%$ & $75 \%$ & $63 \%$ \\
\hline K & $100 \%$ & $100 \%$ & $100 \%$ & $100 \%$ & $0 \%$ & $100 \%$ & $83 \%$ \\
\hline L & $100 \%$ & $100 \%$ & $67 \%$ & $100 \%$ & $0 \%$ & $83 \%$ & $75 \%$ \\
\hline M & $100 \%$ & $100 \%$ & $100 \%$ & $50 \%$ & $50 \%$ & $75 \%$ & $79 \%$ \\
\hline N & $100 \%$ & $100 \%$ & $0 \%$ & $100 \%$ & $0 \%$ & $100 \%$ & $67 \%$ \\
\hline General & $95 \%$ & $98 \%$ & $61 \%$ & $82 \%$ & $46 \%$ & $72 \%$ & $76 \%$ \\
\hline
\end{tabular}

Tabla 8. Evaluación de aspectos relacionados calificación semanal de subcontratistas.

\begin{tabular}{|c|c|c|c|c|c|}
\hline Constructora & $\begin{array}{c}\text { Socialización de la } \\
\text { calificación semanal } \\
\text { de subcontratistas }\end{array}$ & $\begin{array}{c}\text { Evaluación de todas las } \\
\text { variables para calificar } \\
\text { el desempeño }\end{array}$ & $\begin{array}{c}\text { Calificación cuantitativa } \\
\text { o cualitativa }\end{array}$ & $\begin{array}{c}\text { Existencia } \\
\text { de cartelera } \\
\text { de desempeño }\end{array}$ & Promedio \\
\hline A & $0 \%$ & $33 \%$ & $0 \%$ & $33 \%$ & $17 \%$ \\
\hline B & $50 \%$ & $100 \%$ & $0 \%$ & $100 \%$ & $63 \%$ \\
\hline C & $100 \%$ & $100 \%$ & $0 \%$ & $75 \%$ & $69 \%$ \\
\hline D & $100 \%$ & $100 \%$ & $0 \%$ & $100 \%$ & $75 \%$ \\
\hline E & $50 \%$ & $0 \%$ & $0 \%$ & $100 \%$ & $38 \%$ \\
\hline F & $100 \%$ & $100 \%$ & $0 \%$ & $100 \%$ & $75 \%$ \\
\hline G & $50 \%$ & $50 \%$ & $0 \%$ & $50 \%$ & $38 \%$ \\
\hline H & $33 \%$ & $33 \%$ & $0 \%$ & $33 \%$ & $25 \%$ \\
\hline I & $100 \%$ & $100 \%$ & $100 \%$ & $100 \%$ & $100 \%$ \\
\hline J & $50 \%$ & $0 \%$ & $0 \%$ & $50 \%$ & $25 \%$ \\
\hline K & $100 \%$ & $100 \%$ & $0 \%$ & $100 \%$ & $75 \%$ \\
\hline L & $100 \%$ & $67 \%$ & $67 \%$ & $67 \%$ & $75 \%$ \\
\hline M & $100 \%$ & $100 \%$ & $0 \%$ & $100 \%$ & $75 \%$ \\
\hline N & $100 \%$ & $100 \%$ & $0 \%$ & $100 \%$ & $75 \%$ \\
\hline General & $74 \%$ & $70 \%$ & $12 \%$ & $79 \%$ & $59 \%$ \\
\hline
\end{tabular}

quienes en teoría participan semanalmente de la reunión a corto plazo, hay una noción un poco más acertada, sin embargo, hay ambigüedad en la mayoría de las respuestas; en los profesionales de la obra, quienes son partícipes de los tres niveles de planificación, aunque hay una mayor claridad en el tema, aún se encuentran muchos casos en los que el concepto es muy vago.
La capacitación inicial acerca de la herramienta muestra un comportamiento similar (ver Figura 2): El porcentaje de profesionales de obra que reciben Capacitaciones formales es significativamente mayor que el de los maestros y subcontratistas, y el porcentaje de estos últimos, es a su vez mayor que el del personal operativo, caso en el que hay 


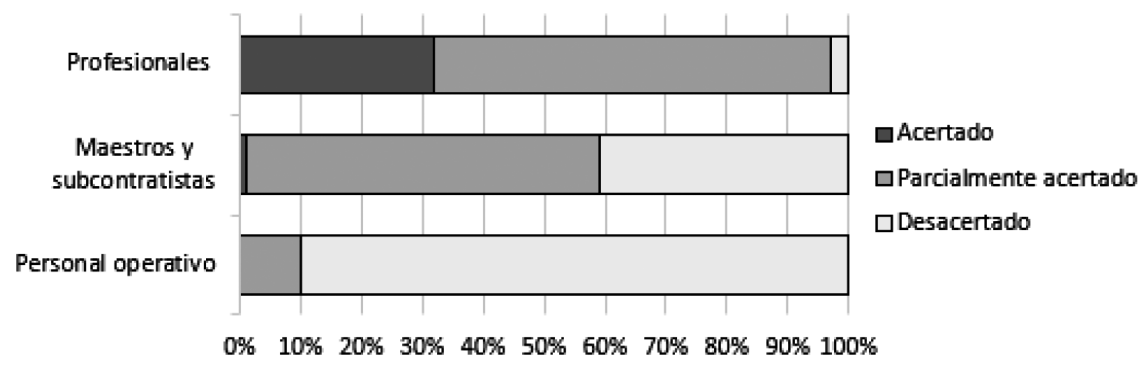

Figura 1. Definición acertada acerca de la definición del SUP.

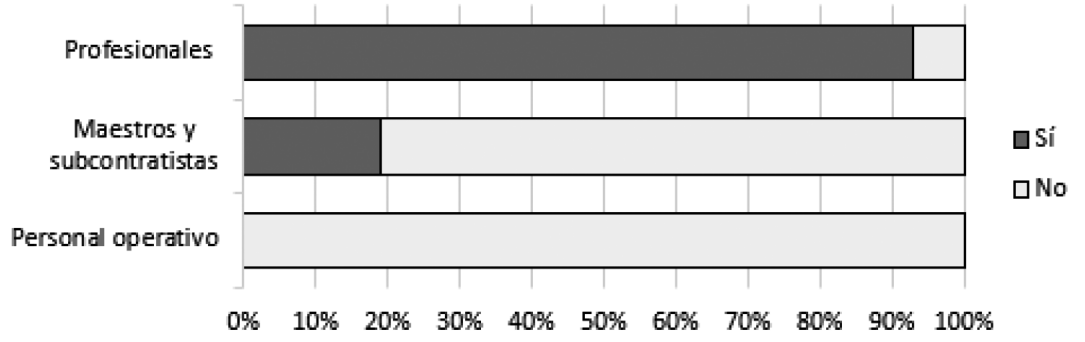

Figura 2. Formalidad de la capacitación inicial.

carencia casi absoluta de capacitaciones formales sobre el SUP.

Respecto al interrogante acerca de la CNC más frecuente, se muestran en la Figura 3 las respuestas con su porcentaje de ocurrencia dentro de cada grupo de encuestados. La CNC más frecuente registrada entre los profesionales se relaciona con la mano de obra, con un $48 \%$ del total de respuestas; para los maestros y subcontratistas, las CNC relacionadas con la mano de obra y los materiales, son las más comunes, con un $20 \%$ de ocurrencia cada una. En

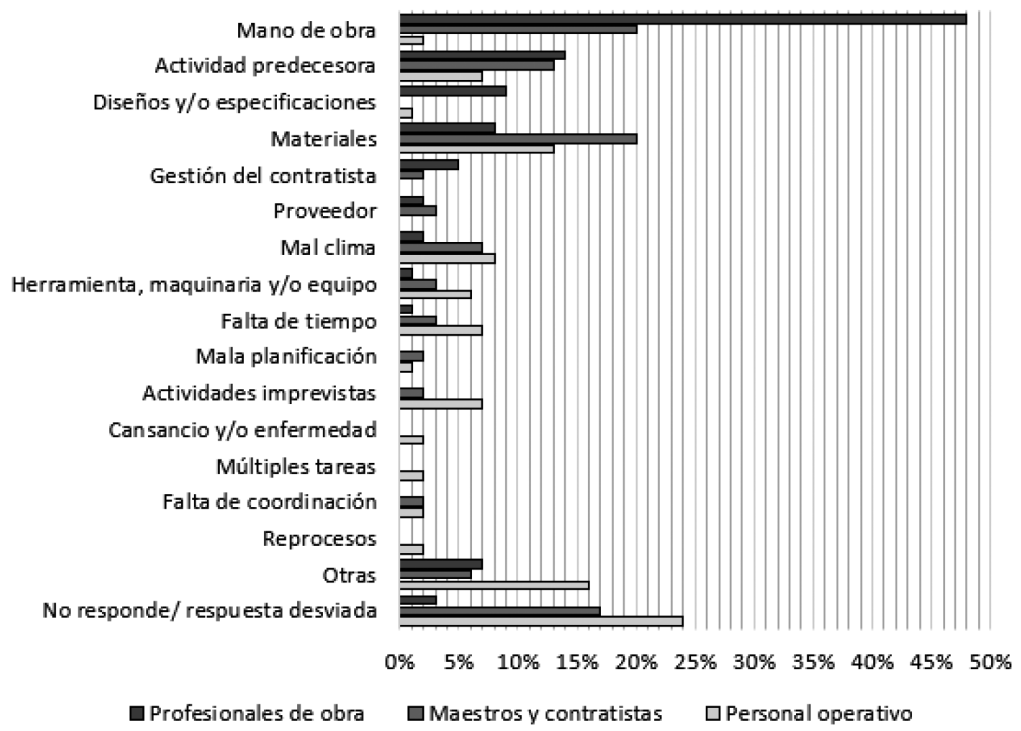

Figura 3. CNC más frecuentes según el grupo de encuestados. 
el caso del personal operativo, las $\mathrm{CNC}$ relativas a los materiales son las más habituales, con un $13 \%$ dentro de la muestra.

Respecto a los aspectos generales de planificación, la tendencia es similar al apartado de noción de la herramienta, los profesionales de obra afirman tener un conocimiento de la planificación de la obra mucho más sólido que los demás tipos de encuestados. Es habitual en los maestros y subcontratistas el desconocimiento de las fechas de entrega y la programación general de la obra, más aún en el personal operativo. Respecto a si se habla o no acerca de las CNC y si se buscan soluciones para evitar recurrencia de las mismas, la gran mayoría de asistentes a la reunión de planificación semanal aseguraron hacerlo, sin embargo, esto se contrasta con las observaciones del autor en el proceso de visita a las reuniones en las obras, donde estas prácticas no se observaron con frecuencia (ver Figura 4).

En relación con su apreciación respecto al sistema actual de planificación y control en obra, la mayoría de encuestados se encuentran satisfechos, pues catalogan dicho sistema como "bueno" (ver Figura 5).

Respecto al factor humano se preguntó al grupo de maestros y subcontratistas y al personal operativo si se sentían o no escuchados dentro de la obra, y si la constructora exaltaba su labor cuando su equipo de trabajo cumplía con las labores planificadas. Ante el primer interrogante las respuestas fueron en su mayoría positivas (ver Figura 6), especialmente en el grupo de maestros y subcontratistas, quienes tienen el papel de últimos planificadores en las reuniones semanales.

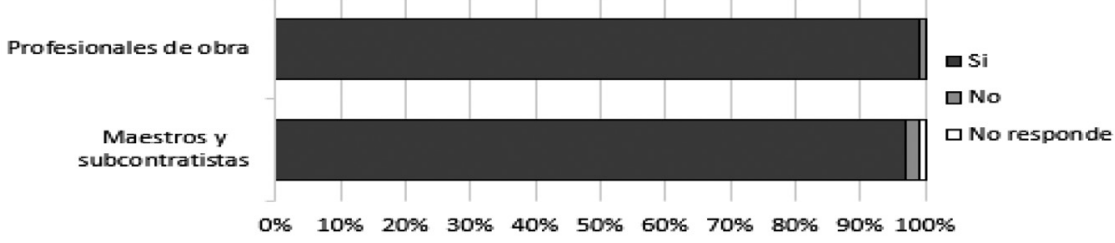

Figura 4. Discusión acerca de las CNC en la reunión semanal.

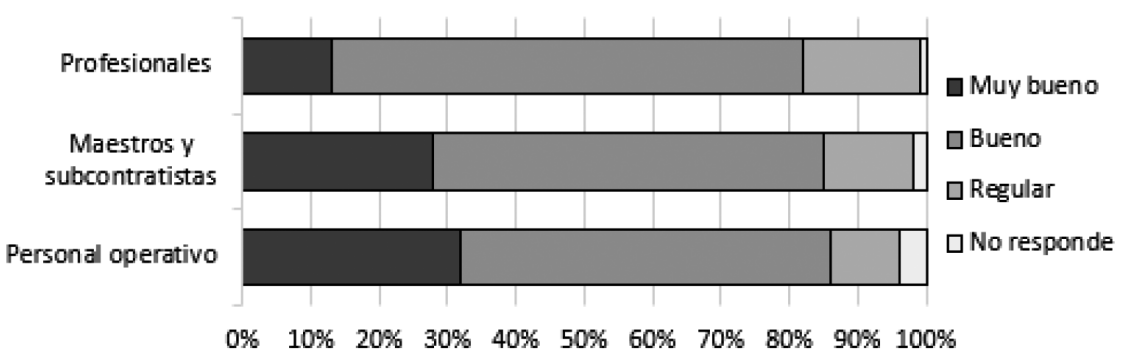

Figura 5. Percepción del personal sobre el sistema de planificación y control en obra.

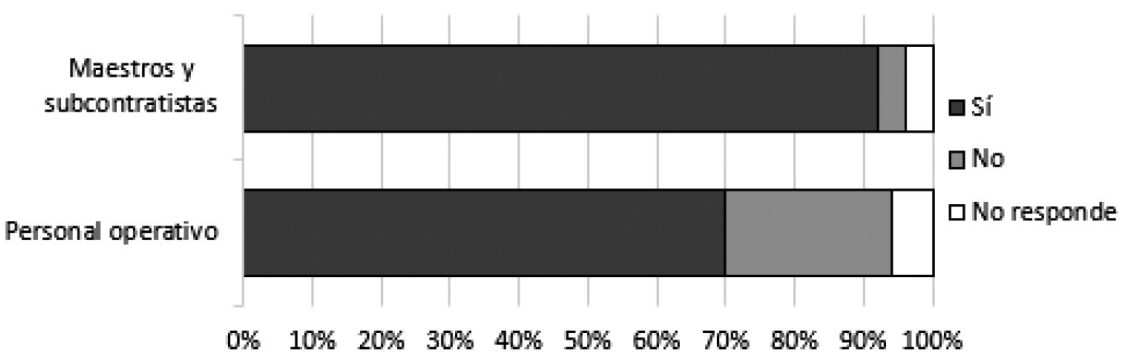

Figura 6. Consideración del personal sobre la escucha que recibe en obra. 
En cuanto al reconocimiento por el deber cumplido, se obtuvieron respuestas similares en ambos grupos de encuestados (ver Figura 7), el 45\% de personas afirmó tener reconocimientos como estímulos económicos, refrigerios o felicitaciones, el resto de encuestados afirmó no tener algún tipo de estímulo o no respondieron a la pregunta.

\section{Entrevistas}

Las entrevistas a los cargos de los entrevistados, responsables de dirigir el área Lean en sus empresas, poseían diferentes jerarquías, algunos tenían un perfil gerencial, otros estaban más enfocados al trabajo de campo y otros tenían funciones administrativas y de sitio; asimismo, la antigüedad en su cargo y dentro de la compañía era muy diversa, y tenían como profesión la ingeniería civil, la ingeniería industrial o la arquitectura (ver Tabla 9).
El primer acercamiento de estas compañías al tema de construcción Lean, se dio hace aproximadamente entre dos y diecisiete años, de la mano de algunas universidades colombianas y la Cámara Colombiana de la Construcción (CAMACOL). Sin embargo, la retroalimentación de la metodología y la capacitación al personal nuevo que ingresa a las compañías no se da en todos los casos ni cobija a todo tipo de personal. Se encontró, además, que en un $40 \%$ de las constructoras no existía personal de soporte para la implementación de las herramientas Lean, eran funciones a cargo de los profesionales técnicos de la obra; en el $60 \%$ restante sí se contaba con personal de soporte, sin embargo, de este porcentaje solo un $56 \%$ tenía un encargado Lean por obra, bien fuera profesionales o practicantes, el $46 \%$ restante contaba con uno o varios profesionales que iban rotando por las diferentes obras.

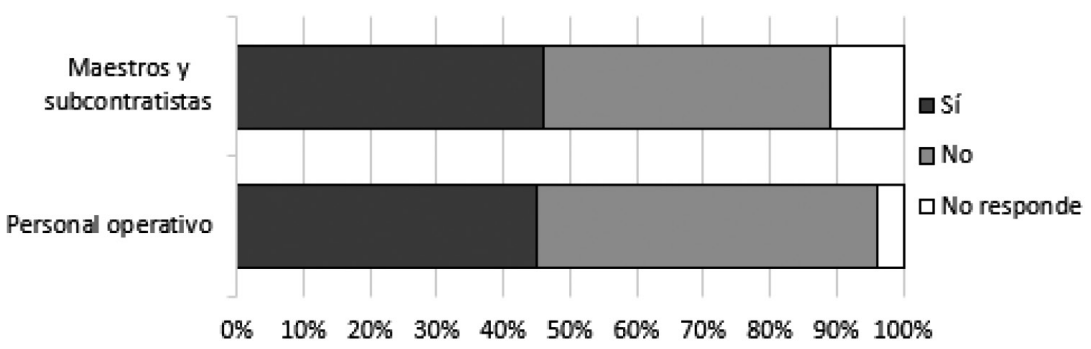

Figura 7. Reconocimiento al personal por el deber cumplido.

Tabla 9. Identificación de los entrevistados.

\begin{tabular}{|c|c|c|c|c|}
\hline \multirow[b]{2}{*}{ Constructora } & \multicolumn{4}{|c|}{ Identificación del encargado del área Lean } \\
\hline & Cargo & $\begin{array}{l}\text { Antigüiedad } \\
\text { en el cargo }\end{array}$ & $\begin{array}{l}\text { Antigüedad en } \\
\text { la empresa }\end{array}$ & Profesión \\
\hline A & Profesional de planeación & 2,5 años & 2,5 años & Ingeniero civil \\
\hline $\mathrm{B}$ & Residente Lean & 6 meses & 6 meses & Ingeniero industrial \\
\hline $\mathrm{C}$ & Coordinador de Lean construction & 2,5 años & 2,5 años & Ingeniero de producción \\
\hline $\mathrm{D}$ & Subdirector de construcción & 6 meses & 16 años & Ingeniero civil \\
\hline $\mathrm{E}$ & Coordinador de gestión integral & 1,25 años & 4 años & Ingeniero industrial \\
\hline $\mathrm{F}$ & Coordinador de productividad del área de innovación & 7 años & 10 años & Ingeniero civil \\
\hline $\mathrm{G}$ & Director de interventoría e interventora & 13 años & - & Arquitectos constructores \\
\hline $\mathrm{H}$ & Director de gestión de proyectos & 1 año & 5 años & Ingeniero civil \\
\hline I & Director de gestión de calidad & 8 años & 8 años & Ingeniero industrial \\
\hline $\mathrm{J}$ & Coordinador de planeación de operaciones & 1,5 años & 4 años & Ingeniero civil \\
\hline $\mathrm{K}$ & Director técnico administrativo & 5 años & 40 años & Ingeniero civil \\
\hline $\mathrm{L}$ & Coordinador Lean construction & 8 meses & 8 meses & Ingeniero industrial \\
\hline $\mathrm{M}$ & Coordinador Lean construction & 1,5 años & 1,5 años & Ingeniero industrial \\
\hline $\mathrm{N}$ & Coordinador Lean construction & 8 años & 8 años & Ingeniero industrial \\
\hline $\mathrm{O}$ & Asesor en implementación Lean & 4 años & No aplica & Ingeniero civil \\
\hline
\end{tabular}


Se han implementado en todos los casos los tres niveles de planificación, sin embargo, en algunas empresas los entrevistados manifiestan tener contrariedades con la planificación intermedia o expresan la falta de relación entre los tres niveles de planificación. Para la realización del plan general o plan maestro, se utilizan dos técnicas de planificación: Diagramas de Gantt o líneas de balance. El software más común para el primer caso es el Microsoft Project, no obstante, se usa también el Gantt Project; para las líneas de balance la mayoría de compañías emplean Microsoft Excel o Vico Software. Respecto a la planificación intermedia, el horizonte de tiempo varía entre 4 y 12 semanas según la compañía. Las reuniones en este nivel de planificación se hacen típicamente cada semana, sin embargo, hay casos en los que es evidente la falta de claridad respecto a este componente del SUP en la compañía o, por lo menos, del entrevistado, pues la periodicidad de las reuniones coincide con el horizonte de tiempo, lo cual no va en línea con el objetivo de revisar continuamente la liberación de restricciones de las actividades. Finalmente, en cuanto a la reunión de planificación semanal, todos los entrevistados coinciden en que en las obras de sus compañías se exponen las CNC de las actividades y se busca la causa raíz de dicho incumplimiento.

Respecto a la opinión acerca de la mejora más significativa, se resaltan principalmente aspectos culturales sobre la actitud de las personas hacia el compromiso, el cumplimiento con la programación, la reducción de tiempos y el paso de la informalidad a la formalidad. Por otro lado, en cuanto a lo que se podría mejorar, sobresale la integración de todas las fases del proyecto, la profundización en la planificación intermedia y software que facilite la elaboración de las líneas de balance. La respuesta a estos dos interrogantes se resume en la Tabla 10 y Tabla 11.

\section{DISCUSIÓN DE RESULTADOS}

Todas las compañías analizadas en el presente estudio han implementado el SUP durante al menos dos años y los encargados del área Lean resaltan, entre otros, las mejoras en el cumplimiento del programa, el ajuste de las programaciones al

Tabla 10. Opinión acerca de la mejora más significativa con implementación del SUP.

\begin{tabular}{|c|c|}
\hline Constructora & ¿Cuál ha sido la mejora más significativa después de la implementación del SUP? \\
\hline A & $\begin{array}{l}\text { "Tuvimos un proyecto en el que se llevó muy juiciosamente la herramienta, una estructura de } \\
\text { cuatro meses la hicimos en tres, nos ahorramos un mes". }\end{array}$ \\
\hline $\mathrm{B}$ & "El ritmo de avance del trabajo del subcontratista". \\
\hline $\mathrm{C}$ & $\begin{array}{l}\text { "La forma de comunicación con el subcontratista, con la reunión semanal se direccionan las } \\
\text { actividades de una manera más formal y controlada". }\end{array}$ \\
\hline $\mathrm{D}$ & "Se han mejorado los cumplimientos de los programas de obra, eso es un hecho". \\
\hline $\mathrm{E}$ & "Las programaciones que estamos buscando son lo más cercano a lo que queremos". \\
\hline $\mathrm{F}$ & "La cultura, aunque lastimosamente se va perdiendo si no se sigue con la misma rigurosidad". \\
\hline $\mathrm{G}$ & "Todo es un orden, el SUP ha permitido tener conocimiento de qué es lo que hay que controlar". \\
\hline $\mathrm{H}$ & $\begin{array}{l}\text { "La reducción de tiempos, de desperdicios y ver que realmente una obra se puede hacer en menor } \\
\text { tiempo y optimizando recursos". }\end{array}$ \\
\hline I & $\begin{array}{l}\text { "Lograr que la gente comprenda que la planificación vale la pena, que el esfuerzo es muy } \\
\text { importante y que no debemos vivir para apargar incendios". }\end{array}$ \\
\hline $\mathrm{J}$ & $\begin{array}{l}\text { "La comunicación formal. Antes toda la comunicación con los subcontratistas era informal, no } \\
\text { teníamos herramientas para actuar legalmente". }\end{array}$ \\
\hline $\mathrm{K}$ & "La participación y sensibilización de la gente hacia el compromiso". \\
\hline $\mathrm{L}$ & $\begin{array}{l}\text { "La disposición de la gente, porque de nada sirve tener unas muy buenas herramientas que te } \\
\text { automatizan todos los procesos si la gente no está convencida de ellas". }\end{array}$ \\
\hline M & $\begin{array}{l}\text { "El pasar de la informalidad a la formalidad, eso es un plus que da el SUP. Dejar de hacer o dar } \\
\text { las directrices de manera informal". }\end{array}$ \\
\hline $\mathrm{N}$ & "La reducción de los tiempos y el cumplimiento de las fechas de entrega de los proyectos" \\
\hline $\mathrm{O}$ & $\begin{array}{l}\text { "Tener datos ciertos sobre los cuales actuar, como las causas de no cumplimiento que le muestran } \\
\text { a uno la realidad". }\end{array}$ \\
\hline
\end{tabular}


Tabla 11. Opinión acerca de los aspectos a mejorar en el sistema de planificación y control.

\begin{tabular}{|c|c|}
\hline Constructora & ¿Qué se podría mejorar a nivel de planificación y control dentro de la empresa? \\
\hline A & "Cambiarles el chip a los viejos, por ejemplo, alguien que ha hecho lo mismo durante 40 años". \\
\hline B & $\begin{array}{l}\text { "Conocer las planificaciones general e intermedia, tener un acceso diario para poder saber dónde } \\
\text { está, cuáles fueron las restricciones en su momento y hoy cuales son". }\end{array}$ \\
\hline $\mathrm{C}$ & $\begin{array}{l}\text { "Las etapas iniciales de concepción del proyecto, todavía se hacen muchos cambios sobre la } \\
\text { marcha". }\end{array}$ \\
\hline $\mathrm{D}$ & "A tighter follow-up of things' of performance and greater control in those results". \\
\hline $\mathrm{E}$ & "Un seguimiento más estricto de los cumplimientos de las cosas, mayor control en esos resultados". \\
\hline $\mathrm{F}$ & $\begin{array}{l}\text { "Lograr integrar todas las fases del proyecto, desde el diseño, la programación, las compras, el } \\
\text { presupuesto, la ejecución". }\end{array}$ \\
\hline $\mathrm{H}$ & "La parte de logística, de entrega de materiales o distribución de materiales al interior es mínima". \\
\hline I & $\begin{array}{l}\text { "Debemos comenzar a ser más proactivos y preventivos... que todos entiendan, que un sistema } \\
\text { de producción en obra depende principalmente de los que están adentro". }\end{array}$ \\
\hline $\mathrm{J}$ & $\begin{array}{l}\text { "La planificación a mediano plazo... la dificultad es cuando uno quiere inmiscuir a diseño, a } \\
\text { contratación, a compras, a todas las personas que intervienen en el proceso". }\end{array}$ \\
\hline K & $\begin{array}{l}\text { "Yo creo que hay que volver a hacer una sensibilización importante en el tema de Lean y es } \\
\text { volver a medir... uno siempre lo que quiere es aumentar el tiempo productivo". }\end{array}$ \\
\hline $\mathrm{L}$ & "La rotación de personal que no me permite realizar una curva de aprendizaje". \\
\hline M & $\begin{array}{l}\text { "Considero que se puede mejorar mucho más el control de las actividades, los volúmenes de } \\
\text { trabajo son altos". }\end{array}$ \\
\hline $\mathrm{N}$ & $\begin{array}{l}\text { "De pronto contar con un software más fácil que el Excel para hacer las líneas de balance. Los } \\
\text { software son costosos". }\end{array}$ \\
\hline $\mathrm{O}$ & $\begin{array}{l}\text { "Pienso que algo que nos haga la línea de balance, un software facilitaría mucho la labor, sería } \\
\text { algo positivo". }\end{array}$ \\
\hline
\end{tabular}

desempeño real de los proyectos, la conciencia de compromiso de las personas, la comunicación formal con subcontratistas, el orden en el sitio de trabajo y la cultura de planificación en el personal involucrado. Sin embargo, aún no se han establecido sistemas de indicadores de desempeño que relacionen directamente la implementación de SUP con los resultados finales del proyecto; además, se encuentran numerosas falencias en la etapa de aplicación y sostenimiento de la metodología que no permiten obtener el máximo beneficio de esta.

A partir de la información recopilada en campo, la cual fue posteriormente tabulada, codificada y analizada, se puso de manifiesto la falta de interiorización del sistema, desde los encargados del área Lean o productividad, hasta los subcontratistas y personal operativo, último eslabón de la cadena de planificación. Los principios del SUP deben permear la cultura de la empresa, desde la cabeza de la organización hasta el nivel de la unidad de producción, pues de otra forma el éxito de la herramienta no será el esperado. Asimismo, debe haber articulación entre los tres niveles de planificación, el plan semanal debe estar sincronizado con el plan intermedio, y este a su vez con el plan general, para garantizar un flujo de trabajo continuo que minimice las ineficiencias y disminuya las pérdidas. Respecto a lo anterior, en la mayoría de las constructoras no se encontró correspondencia entre los tres niveles de planificación, puesto que en muchos casos había aún restricciones al momento de programar las asignaciones a corto plazo y, en algunas obras, inclusive, la programación intermedia se hacía durante la misma reunión semanal de forma improvisada, preguntando a los subcontratistas qué actividades deberían realizarse. Estos casos puntuales dejan ver que los profesionales de obra, quienes están encargados de velar por el cumplimiento del programa de obra, no tienen claro en qué punto está el proceso constructivo y qué se debe y puede hacer a la luz de la planificación intermedia, lo cual demuestra falta de apropiación de la metodología. Una implementación pobre del nivel intermedio de planificación y el vínculo débil entre dicho nivel y la ejecución de asignaciones, coincide con los reportes en la literatura de Ballard [27], Hamzeh et al. [25], Hamzeh et al. [26], Shang y Pheng [14] y Khanh y Kim [18]. 
Los vacíos en la noción de la herramienta se hallaron más marcados en los maestros y subcontratistas, y, de forma incuestionable, en el personal operativo. A través de las encuestas fue posible advertir un desconocimiento generalizado respecto a la definición del sistema, a pesar de que el SUP era la metodología de planificación y control empleada en todas las obras, e incluso en algunos casos era común para todo el equipo de trabajo escuchar al interior de los proyectos la expresión "Last Planner". En línea con lo anterior, se encontró que en muchos casos utilizaban dicha expresión para referirse únicamente a la reunión de planificación semanal o a la calificación de subcontratistas, o se hacía referencia a la reunión de planificación a corto plazo como "el PAC". Aunque en todas las obras visitadas se realizaba la reunión semanalmente, en algunas solo se conocía con su nombre dentro de los profesionales de obra; entre los maestros y subcontratistas, que en la mayoría de las constructoras participaban de dicha reunión, solían utilizarse otras expresiones. La poca familairidad con los conceptos y estrategias del SUP han sido reportados en otros casos de estudio por Liu et al. [47], Ballard et al. [9] y Friblick et al. [35].

En relación con los aspectos generales de planificación indagados, hay puntos en los que surge el contraste entre lo que el autor observó en las visitas a las obras y el resultado de las encuestas en los tres grupos de encuestados. Especialmente se encontró falta de correspondencia entre el registro de restricciones en la planificación intermedia y la discusión acerca de las CNC. A pesar de que en las encuestas la mayoría de profesionales de obra, maestros y subcontratistas afirmaron cumplir con estos dos puntos, durante la observación no participante se encontró con frecuencia falencia al respecto; esto puede asociarse con la falta de madurez para realizar autodiagnóstico del desempeño en las compañías. En muchas ocasiones no se discutía acerca de las CNC, el encargado de dirigir la reunión dejaba el registro dentro de la plantilla del software empleado, pero no había socialización durante la reunión. Las CNC eran, generalmente, muy ambiguas, si se registraba "demora proveedor" no se especificaba qué proveedor o el motivo de la demora, asimismo, en causas como falta de material o de herramienta y equipo o mano de obra, no se hacía la salvedad del responsable. El diálogo en torno a las CNC debe ser uno de los puntos fuertes de la reunión de planificación semanal, asimismo el ahondamiento en la causa raíz que genera el incumplimiento, con miras a poder intervenir oportunamente para que no haya recurrencia. Un análisis pobre de la causa raíz de los incumplimientos ha sido reportado también por Kim y Ballard [48], Hamzeh et al. [25] and Mcconaughy and Shirkey [23].

Del proceso de observación no participante, fue posible concluir que no hay dependencia con significancia estadística entre los años implementando herramientas Lean y el buen desempeño en los principales puntos observados: Desarrollo de la reunión, aspectos de formalidad durante el curso de la misma y aspectos relacionados con los subcontratistas. Asimismo, no hay correlación fuerte en los aspectos observados, es decir, no siempre que en una constructora se desarrollaba de forma adecuada la reunión se tenían en cuenta aspectos de formalidad durante la misma o existía un sistema de calificación al subcontratista eficiente; en general se encontraron compañías con fortalezas en algunos puntos y debilidades en otros.

\section{CONCLUSIONES}

El SUP, herramienta de planificación y control de la construcción concebida dentro de los principios de la filosofía Lean, se comenzó a difundir hace aproximadamente diez años en el gremio constructor colombiano y aunque en teoría las compañías han trabajado en su implementación, aún se encuentran falencias en la etapa de aplicación y sostenimiento en el tiempo. A partir de la información recopilada en campo, la cual fue posteriormente tabulada y analizada, se pone de manifiesto la falta de interiorización del sistema, desde los encargados del área Lean o productividad, hasta los subcontratistas y personal operativo, último eslabón de la cadena de planificación. Los principios del SUP deben permear la cultura de la empresa, desde la cabeza de la organización hasta el nivel de la unidad de producción, pues de otra forma el éxito de la herramienta no será el esperado. Asimismo, debe haber articulación entre los tres niveles de planificación, el plan semanal debe enmarcarse en el plan intermedio, y este a su vez dentro del plan general, para garantizar un flujo de trabajo continuo.

En relación con las $\mathrm{CNC}$, los autores sugieren evitar aquellas que estén definidas como un simple 
sustantivo, procurando siempre acompañar la expresión de una palabra, bien sea indicadora de acción o cualidad, que permita precisarla, además se recomienda indagar con la técnica "cinco por qué" acerca del incumplimiento, herramienta sistemática de preguntas introducida por Taiichi Ohno en Toyota y utilizada durante la fase de análisis de problemas para buscar las posibles causas principales de un problema y habituar a las personas a meterse en él y a encontrar respuesta a las preguntas [49]. El equipo debe preguntarse por qué se dio el incumplimiento y sobre la respuesta volver a preguntarse por qué, y así sucesivamente, hasta encontrar la causa raíz que originó el incumplimiento y actuar sobre ella para eliminarla, de tal forma que se minimice su aparición en futuras asignaciones.

Finalmente, cabe destacar que a pesar del esfuerzo por crear cultura y el uso de herramientas que faciliten la transparencia a través del gerenciamiento visual, las empresas de construcción colombianas aún no han establecido sistemas de indicadores de rendimiento que puedan relacionar directamente la implementación del SUP con los resultados finales del proyecto. Además, la alta rotación de personal en el sector de la construcción, no solo a nivel de mano de obra, maestros y subcontratistas, sino a nivel de profesionales de la construcción, afecta directamente la implementación y la propiedad del SUP, ya que hay un cambio constante de personal y la curva de aprendizaje debe comenzar de nuevo. Lo anterior pone de manifiesto la importancia de que dentro de la compañía haya transferencia del conocimiento a través de una capacitación inicial formal, benchmarking interno constante y retroalimentación periódica de la metodología, esto motivado principalmente por tres aspectos: La alta rotación del personal en el medio, las relaciones de interdependencia propias del proceso constructivo y el deseo de que la filosofía haga parte de la cultura de todos los involucrados.

Los autores dejan una invitación abierta a las empresas colombianas a fortalecer las capacitaciones teóricas acerca del SUP y a retroalimentar periódicamente a todo el equipo de trabajo, pues el proceso de implementación debe estar acompañado de una base conceptual sólida que permita dar continuidad en el tiempo a la herramienta. Además, se resalta la importancia de que pueda determinarse el impacto del SUP en término de cumplimiento de plazos y su relación directa con el ahorro en términos monetarios, asimismo, que haya trazabilidad en los tres niveles del proceso de planificación y en el desempeño de los subcontratistas por medio de indicadores de gestión. Se motiva finalmente a la investigación y documentación acerca del grado de implementación y funcionamiento del SUP en las compañías, las dificultades y casos de éxito en su aplicación, esto con el fin de generar un banco de conocimientos más amplio que incentive la comparación de desempeño y un mejoramiento continuo de los profesionales y personal involucrado en el sector constructor.

\section{REFERENCIAS}

[1] L. Koskela. "Application of the new production philosophy to construction". Stanford University. 1992.

[2] G. Ballard. "The last planner system of production control". Doctor of Philosophy School of Civil Engineering, Faculty of Engineering, University of Birmingham. Salford, U.K. 2000.

[3] A.D. Rodríguez, L.F. Alarcón and E. Pellicer. "La gestión de la obra desde la perspectiva del último planificador". Revista de Obras Públicas. N 3518. 2011. ISSN: 0034-8619.

[4] CAMACOL. "Tendencias de la construcción. Economía y coyuntura sectorial”. Bogotá D.C, Colombia. 2018.

[5] Departamento Administrativo Nacional de Estadística. "Indicadores Económicos Alrededor de la Construcción - IEAC. I trimestre de 2018”. Boletín Técnico. Departamento Administrativo Nacional de Estadística - DANE. Bogotá, Colombia. 27 de junio de 2018.

[6] G. Ballard. "Improving work flow reliability". 7th Annual Conference of the International Group for Lean Construction. Berkeley, USA. 1999.

[7] M.F. Hoyos y L.F. Botero. "Evolución e impacto mundial del last planner system: Una revisión de la literatura". Ingeniería y Desarrollo. Vol. 36. 2018.

[8] I. Adamu and G. Howell. "Applying last planner in the nigerian construction industry". 20th Annual Conference of the International Group for Lean Construction. San Diego, USA. 2012. 
[9] G. Ballard, M. Casten and G. Howell. "PARC: A case Study". 4th Annual Conference of the International Group for Lean Construction. Birmingham, UK. 1996.

[10] Y.-W. Kim and J.-W. Jang. "Case study: An application of last planner to heavy civil construction in Korea". 13th Annual Conference of the International Group for Lean Construction. Sydney, Australia. 2005.

[11] L.F. Alarcón, S. Diethelmand, O. Rojo and R. Calderón. "Assessing the impacts of implementing lean construction". Revista Ingeniería de Construcción. Vol. 23, pp. 26-33. 2008. DOI: 10.4067/ S0718-50732008000100003

[12] L.F. Alarcón, S. Diethelmand and O. Rojo. "Collaborative Implementation of lean planning systems in chilean construction companies". 10th Annual Conference of the International Group for Lean Construction. Gramado, Brazil. 2002.

[13] A. Alsehaimi, P. Tzortzopoulos and L. Koskela. "Last planner System- Experiences From Pilot Implementation in the Middle East". 17th Annual Conference of the International Group for Lean Construction. Taipei, Taiwan. 2009.

[14] G. Shang and L. Pheng. "The last planner system in China's construction industry - A SWOT analysis on implementation". International Journal of Project Management. Vol. 32, pp. 1260-1272. 2014. DOI: 10.1016/j. ijproman.2014.01.002

[15] F. Hamzeh, J. Kallassy, M. Lahoud and R. Azar. "The first extensive implementation of lean and LPS in Lebanon: Results and reflections". 24th Annual Conference of the International Group for Lean Construction. Boston, USA. 2016.

[16] O. Ahiakwo, D. Oloke, S. Suresh and J. Khatib. "A case study of last planner system implementation in Nigeria". 21st Annual Conference of the International Group for Lean Construction. Fortaleza, Brazil. 2013.

[17] J. Fuemana, T. Puolitaival and K. Davies. "Last planner system a step towards improving the productivity of New Zealand construction". 21st Annual Conference of the International Group for Lean Construction. Fortaleza, Brazil. 2013.
[18] H. Khanh and S. Kim. "A survey on production planning system in construction projects based on last planner system". KSCE Journal of Civil Engineering. Vol. $20 \mathrm{~N}^{\circ}$ 1, pp. 1-11.2015. DOI: 10.1007/ s12205-015-1412-y

[19] V. Priven and R. Sacks. "Effects of the last planner system on social networks among construction trade crews". Journal of Construction Engineering and Management. Vol. $141 \mathrm{~N}^{\circ}$ 6, pp. 1-10. 2015. DOI: 10.1061/ (ASCE)CO.1943-7862.0000975

[20] F. Cerveró-Romero, P. Napolitano, E. Reyes and L. Teran. "Last planner system and lean approach process experiences from implementation in México". 20th Annual Conference of the International Group for Lean Construction. Fortaleza, Brazil. 2013.

[21] P. Arroyo and O. Valladares. "Last planner system: Implementation, evaluation and comparison of results in the construction of a social housing project in Chile". 24th Annual Conference of the International Group for Lean Construction. Boston, USA. 2016.

[22] C. Mejía-Plata, J.S. Guevara-Ramírez, D. F. Moncaleano-Novoa, M.C. Londoño-Acevedo, J.S. Rojas-Quintero and J.L. Ponz-Tienda. "A route map for implementing last planner system in Bogota, Colombia". 24th Annual Conference of the International Group for Lean Construction. Boston, USA. 2016.

[23] T. Mcconaughy and D. Shirkey. "Subcontractor collaboration and breakdowns in productionthe effects of varied lps implementation". 21st Annual Conference of the International Group for Lean Construction. Fortaleza, Brazil. 2013.

[24] E. Johansen and G. Porter. "An experience of introducing last planner into a UK construction project". 11th Annual Conference of the International Group for Lean Construction. Virginia, USA. 2003.

[25] F. Hamzeh, G. Ballard and I. Tommelein. "Improving construction work flow - the connective role of lookahead planning". 16th Annual Conference of the International Group for Lean Construction. Manchester, UK. 2008.

[26] F. Hamzeh, G. Ballard and I. Tommelein. "Rethinking lookahead planning to optmize construction Worflow". Lean Construction Journal, pp. 15-34. 2012. 
[27] G. Ballard. "Lookahead planning. The missing link in production control". 5th Annual Conference of the International Group for Lean Construction. Gold Coast, Australia. 1997.

[28] A.S. Itri. "Last planner, look ahead, PPC: A driver to the site operations". 6th Annual Conference of the International Group for Lean Construction. Guarujá, Brazil. 1998.

[29] M. Fiallo and V.H. Revelo. "Applying the last planner control system to a construction project: A case study in Quito, Ecuador". 10th Annual Conference of the International Group for Lean Construction. Gramado, Brazil. 2002.

[30] A. Soares, M. Bernardes and C. Formoso. "Improving the production planning and control system in a building company: Contributions After Stabilization". 10th Annual Conference of the International Group for Lean Construction. Gramado, Brazil. 2002.

[31] L.F. Botero and M.E. Álvarez. "Last planner, un avance en la planificación y control de proyectos de construcción". Ingeniería y Desarrollo. No 17 , pp. 148-159. 2005.

[32] U.H. Issa. "Implementation of lean construction tecniques for minimizing the risks effect on project construction time". Alexandria Engineering Journal. Vol. 52, pp. 697-704. 2013. DOI: 10.1016/ j. aej. 2013.07.003

[33] J.J. Ochoa. "Reducing plan variations in delivering sustainable building projects". Journal of Cleaner Production. Vol. 85, pp. 276-288. 2014. DOI: 10.1016/j.jclepro. 2014.01.024

[34] J.A. Junior, A. Scola and S.A. Itri. "Last planner as a site operations tool". 6th Annual Conference of the International Group for Lean Construction. Guarujá, Brazil. 1998.

[35] F. Friblick, V. Olsson and J. Reslow. "Prospects for Implementing last planner in the construction industry". 17th Annual Conference of the International Group for Lean Construction. Taipei, Taiwan. 2009.

[36] D. Viana, C. Mota, C. Formoso, M. Echeveste, M. Peixoto and C. Rodrigues. "A survey on the last planner system- impacts and difficulties for implementation in brazilian companies". 18th Annual Conference of the
International Group for Lean Construction. Haifa, Israel. 2010.

[37] L.F. Alarcón, J.L. Salvatierra and J.A. Letelier. "Using last planner indicators to identify early signs of project performance". 22nd Annual Conference of the International Group for Lean Construction. Oslo, Noruega. 2014.

[38] H. Hicham, C. Taoufiq, and S. Aziz. "Last planner system: Implementation in a Moroccan construction project". 24th Annual Conference of the International Group for Lean Construction. Boston, USA. 2016.

[39] B.W. Wambeke, L. Min and S.M. Hsiang. "Using last planner and a risk assessment matrix to reduce variation in mechanical related construction tasks". Journal of Construction Engineering and Management. Vol. $138 \mathrm{~N}^{\circ}$ 4, pp. 491-498. 2012.

[40] M. Rusell, M. Liu, G. Howell and S. Hsiang. "Case studies of the allocation and reduction of time buffer through use of the last planner system". Journal of Construction Engineering and Management. Vol. $141 \mathrm{~N}^{\circ} 2.2014$. DOI: 10.1061/(ASCE) CO.1943-7862.0000900

[41] A. Strauss and J. Corbin. "Bases de la investigación cualitativa. Técnicas y procedimientos para desarrollar la teoría fundamentada". Editorial Universidad de Antioquia. Medellín, Colombia. 2002.

[42] I.D. Toro and R.D. Parra. "Fundamentos epistemológicos de la investigación y la metodología de la investigación cualitativa/ cuantitativa". Fondo Editorial Universidad EAFIT. Medellín, Colombia. 2010.

[43] S. Perelló. "Metodología de la investigación social”. Dykinson. Madrid, España. ISBN: 978-84-9849-792-2. 2011.

[44] R. Rojas. "Guía para realizar investigaciones sociales". Plaza y Valdés Editores. México D.F., México. ISBN: 968-856-262-5. 2013.

[45] R. Guber. "La etnografía. Método, campo y reflexividad". Grupo Editorial Norma. Bogotá, Colombia. ISBN: 958-04-6154-6. 2001.

[46] L.E. Alonso. "La mirada cualitativa en sociología. Una aproximación interpretativa". Fundamentos. Madrid, España. 2003.

[47] M. Liu, G. Ballard and W. Ibbs. "Work flow variation and labor productivity. Case study". Journal of Construction Engineering 
and Management, pp. 236-242. 2011. DOI: 10.1061/(ASCE)ME. 1943-5479. 0000056

[48] Y.-W. Kim and G. Ballard. "Management thinking in the earned value method system and the last planner system". Journal of Management in Engineering. Vol. 26
$\mathrm{N}^{\mathrm{o}}$ 4, pp. 223-228. 2010. DOI: 10.1061 I_ASCE_ME.1943-5479.0000026

[49] A. Galgano. "Las tres revoluciones. Caza del desperdicio: Doblar la productividad con la LEAN production". Ediciones Díaz de Santos. Madrid, España. 2004. 